\title{
O EFEITO DO USO DE ANTIOXIDANTES NA PREVENÇÃO E TRATAMENTO DA NEUROPATIA DIABÉTICA NO SISTEMA NERVOSO ENTÉRICO
}

\author{
Paulo Emilio Botura Ferreira ${ }^{1}$ \\ Mônica de Oliveira Belém² \\ Juliano Yasuo $\mathrm{Oda}^{3}$
}

FERREIRA, P. E. B.; BELÉM, M. de O.; ODA, J. Y. O efeito do uso de antioxidantes na prevenção e tratamento da neuropatia diabética no sistema nervoso entérico. Arq. Cienc. Saúde UNIPAR, Umuarama, v. 19, n. 2, p, 115-123, maio/ago. 2015.

\begin{abstract}
RESUMO: O Diabetes mellitus (DM) é um distúrbio metabólico, complexo e de etiologia múltipla. Esta doença causa prejuízos aos sistemas vasculares e nervosos que se manifestam na forma de macro e microangiopatias e neuropatias diabéticas com alta incidência e prevalência na população mundial. Entre as neuropatias existentes, a neuropatia autonômica afeta o trato gastrointestinal (TGI) e caracteriza-se por alterações degenerativas em componentes do sistema nervoso entérico, como neurônios e células gliais. Consequentemente, ocorrem modificações na secreção e motilidade do TGI que são responsáveis por sintomas comuns da doença, tais como náuseas, inchaço, dor abdominal, diarreia, entre outros. Estudos experimentais e clínicos sugerem que o estresse oxidativo esteja envolvido na patogênese e na progressão da neuropatia diabética autonômica no sistema nervoso entérico. Assim, compostos antioxidantes que previnem a formação e/ ou neutralizam os radicais livres oriundos do estresse oxidativo podem ter um papel relevante no tratamento das complicações neurológicas do diabetes mellitus. Neste artigo, realizou-se uma revisão da literatura sobre os principais aspectos, quadro clínico e patogênese do DM e a ocorrência da neuropatia diabética autônoma no sistema nervoso entérico, abordando as principais pesquisas que empregaram antioxidantes para prevenir e/ou tratar os danos neurológicos que são consequências desta patologia.

PALAVAS-CHAVE: Antioxidantes; Sistema nervoso entérico; Diabetes Mellitus; Neuropatia diabética autonômica.
\end{abstract}

\section{THE USE OF ANTIOXIDANTS IN THE PREVENTION AND TREATMENT OF DIABETIC NEUROPATHY IN THE ENTERIC NERVOUS SYSTEM}

\begin{abstract}
S: Diabetes mellitus (DM) is a metabolic complex disorder of multiple etiology. This disease causes damage to the vascular and nervous systems that are seen as macro- and microangiopathies and diabetic neuropathies, with high incidence and prevalence in the world population. Among the existing neuropathies, the autonomic neuropathy affects the gastrointestinal (GI) tract and is characterized by degenerative changes in components of the enteric nervous system, such as neurons and glial cells. Accordingly, changes occur in secretion and motility of the GIT, which are responsible for common symptoms, such as nausea, bloating, abdominal pain, diarrhea, among others. Experimental and clinical studies suggest that oxidative stress is involved in the pathogenesis and progression of autonomic diabetic neuropathy in the enteric nervous system. Thus, antioxidant compounds that prevent the formation and/or neutralize free radicals derived from oxidative stress may play a role in the treatment of neurological complications of diabetes mellitus. In this article, a literature review is performed on the main aspects of the clinical presentation and the pathogenesis of diabetes and autonomous diabetic neuropathy in the enteric nervous system. It also addresses the main research employing antioxidants to prevent and/or treat neurological damages that are consequences of this disease.
\end{abstract}

KEYWORDS: Antioxidants; Enteric nervous system; Diabetes Mellitus; Autonomic diabetic neuropathy.

\section{Introdução}

O Diabetes mellitos (DM) é um distúrbio metabólico, crônico, complexo e de etiologia múltipla que afeta 243 milhões de pessoas em todo o mundo (OLMOS et al., 2012) e segundo projeções atuais, poderá afetar 439 milhões de indivíduos em 2030, causando forte impacto na economia mundial por necessitar de investimentos do setor público (FARAG; GABALLA, 2011). No Brasil alguns dados relatam que até 2025, o país deverá ter 17,6 milhões de diabéticos, duas vezes mais do que os dados atuais que apontam uma população de oito milhões de portadores (BAZZOTE, 2010). Suas principais manifestações são caracterizadas pela hiperglicemia resultante de defeitos na secreção (tipo I) e/ ou ação da insulina (tipo II) pelas células beta do pâncreas (ASSOCIATION, 2011). Entre os portadores do DM,
30 milhões sofrem de alguma forma de neuropatia diabética (SAID, 2007), sendo considerada uma das complicações mais frequentes e desconfortáveis da doença em ambos os tipos de DM (SIMA, 2003).

Entre tais neuropatias, a neuropatia diabética autonômica pode comprometer o trato gastrintestinal (TGI), causando prejuízos na função gástrica (sensorial e motora) e anormalidades na secreção hormonal deste órgão (CLARKE; EWING; CAMPBELL, 1979; FURNESS, 2005). Adicionalmente, estas alterações, por sua vez, estão associadas a desordens de motilidade gastrointestinal, responsáveis por complicações comuns da doença como náuseas, inchaço, dor abdominal, diarreia, constipação e retardo no esvaziamento gástrico (BYTZER et al., 2001; SAMSOM et al., 2003), afetando aproximadamente $75 \%$ dos pacientes (CHANDRASEKHARAN et al., 2011). A patogênese destas alte-

DOI: https://doi.org/10.25110/arqsaude.v19i2.2015.5432

${ }^{1}$ Biólogo, Doutor em Ciências Biológicas (biologia celular e molecular) e Pós-doutorando do Programa de Pós Graduação em Patologia Experimental pela Universidade Estadual de Londrina (UEL); Laboratório de Neurogastroenterologia.

${ }^{2}$ Biomédica, Mestranda do Programa de Pós-Graduação em Patologia Experimental pela Universidade Estadual de Londrina (UEL); Laboratório de Neurogastroenterologia (UEL).

${ }^{3}$ Fisioterapeuta, Mestre em Anatomia e Doutorando do Programa de Pós-Graduação em Patologia Experimental pela Universidade Estadual de Londrina (UEL); Laboratório de Neurogastroenterologia (UEL).

Endereço para correspondência: Paulo Emilio Botura Ferreira. pbotura@gmail.com 
rações no TG de indivíduos com DM está sob investigação e o papel do sistema nervoso entérico (SNE) e seus neurotransmissores ganhou importância ao longo dos últimos anos (CHANDRASEKHARAN et al., 2011).

O SNE é composto por um grande número de neurônios e células gliais que formam uma rede em toda a parede do TGI (FURNESS, 2012). Tal sistema é conhecido como "cérebro do intestino" e opera independentemente do sistema nervoso central (HERMES-ULIANA et al., 2013). Dessa forma, o SNE modula funções complexas como a motilidade, a secreção e o fluxo sanguíneo do trato gastrintestinal (FURNESS; KUNZE; CLERC, 1999; PALOMBIT et al., 2013), além de ser um sistema plástico e vulnerável a lesões (HERMES-ULIANA et al., 2013). Assim, doenças que afetam esse sistema, podem alterar as funções gastrointestinais (SRINIVASAN; WILEY, 2000).

De fato, muitos autores têm demonstrado que a neuropatia autonômica causada pelo DM crônico está relacionada à alterações quantitativas e morfométricas no SNE de vários segmentos do TGI de ratos com DM experimental (ZANONI et al., 2003; SHOTTON; ADAMS; LINCOLN, 2007; PEREIRA et al., 2011; LOPES et al., 2012; FERREIRA et al., 2013; HERMES-ULIANA et al., 2013), além de alterar o código neuroquímico e a liberação de neurotransmissores (VOUKALI; SHOTTON; LINCOLN, 2011; FERREIRA et al., 2013). Estas alterações em neurônios do SNE são atribuídas à condição de hiperglicemia do DM que leva a um aumento do estresse oxidativo e redução na capacidade antioxidante das células (BONNEFONT-ROUSSELOT, 2002).

Nesse contexto, múltiplas estratégias terapêuticas têm demonstrado que o tratamento com antioxidantes previne a formação ou neutraliza as espécies radicalares oriundas do estresse oxidativo, minimizando ou evitando as complicações neurológicas do DM (CAMERON; COTTER, 1999; SHIRPOOR et al., 2007). Assim, o objetivo deste estudo é atualizar informações sobre o quadro clínico e a patogênese do DM e da neuropatia diabética autônoma no sistema nervoso entérico bem como elucidar os possíveis mecanismos que justificam o uso de antioxidantes em seu tratamento, abordando também as pesquisas recentes já desenvolvidas que utilizaram estas substâncias.

\section{Desenvolvimento}

Fisiopatologia e quadro clínico do diabetes mellitus e da neuropatia autonômica diabética

De modo geral, o diabetes mellitus é o resultado da deficiência crônica da ação insulínica no organismo, por alguma insuficiência relativa ou absoluta do hormônio insulina (WOLFF, 1970). Esta patologia é pertencente a um grupo de doenças metabólicas caracterizadas por apresentar um estado crônico de hiperglicemia, resultante de defeitos na secreção e/ou ação do hormônio insulina, ocorrendo uma alteração no metabolismo, em especial o do controle dos níveis de açúcar do organismo (OPPENHEIM, 1996; BAZZOTE, 2010). Existem dois tipos principais de DM, as mais comumente encontradas, DM tipo 1, também conhecida como diabetes juvenil que acomete de 10 à $20 \%$ dos casos, e DM tipo 2 ou diabetes tardio responsável por 80 à $90 \%$ dos casos. Es- tes são classificadas com base nas necessidades de insulina (ASSOCIATION, 2011). Algumas pesquisas relatam que as complicações são consequências dos distúrbios metabólicos, sendo que entre estes distúrbios estão a hiperglicemia e a hipoglicemia (ASSOCIATION, 2008; 2011)

A hiperglicemia sanguínea faz com que o excesso de glicose no sangue seja filtrado no glomérulo renal e não totalmente reabsorvido no túbulo renal, fazendo com que a glicose seja eliminada através da urina, acarretando aumento do volume urinário e caracterizando assim a poliúria. Em consequência a esta perda de água e eletrólitos, há uma desidratação hipertônica levando o paciente a ter sede e aumento da ingestão de água, o que caracteriza, por sua vez, a polidpsia (SANT'ANA et al., 1997). Além destas, a hiperglicemia pode gerar uma acentuada quebra de ácidos graxos (lipólise) nos adipócitos, que caem na corrente sanguínea e chegam ao fígado. Uma parcela destes lipídios é resterificada, mas há os que são carregados pela enzima carnitina acil-transferase para o interior da mitocôndria onde serão utilizados pelo ciclo do ácido tricarboxílicos para gerar energia ou serão transformados nos conhecidos corpos cetônicos que são secundariamente usados como substratos energéticos (SANT'ANA et al., 1997). Entretanto, em casos de produção excessiva de corpos cetônicos, o paciente apresentará uma excreção renal acentuada de bases, o que por sua ação osmótica leva à eliminação de água, desenvolvendo sintomas como náuseas e vômitos. A desidratação desenvolve-se também no sistema nervoso central e aí caracteriza-se por coma hiperglicêmico com cetoacidose (WOLFF, 1970; SANT'ANA et al., 1997; ASSOCIATION, 2008; 2011).

O diabetes mellitus possui complicações que podem ser divididas em agudas e crônicas. Entre essas últimas, destacam-se as neuropatias diabéticas que são decorrentes de lesões de fibras nervosas periféricas por alterações vasculares e metabólicas dos neurônios (LIMA, 1993), com comprometimento das áreas inervadas (ARAÚJO, 1996). Como muitas vezes, as neuropatias são sintomáticas, reduzem a qualidade de vida dos portadores de DM, sendo, por este motivo, muito comuns os estudos sobre as alterações morfológicas e funcionais dos nervos somáticos (GREENE; WINEGRAD, 1979; THURSTON et al., 1995; MALONE et al., 1996). Esses estudos também correlacionam a severidade das alterações com a idade do indivíduo, o grau do estado diabético, e a interferência de outros fatores.

Entre as neuropatias que se manifestam no DM, está a neuropatia autonômica que compromete vários sistemas orgânicos. Em nível gastrintestinal, leva a modificações de suas atividades motora, sensorial e reflexa (CLARKE: EWING; CAMPBELL, 1979) manifestadas comuns da doença como náuseas, inchaço, dor abdominal, diarreia, constipação, retardo no esvaziamento gástrico (BYTZER et al., 2001; SAMSOM et al., 2003), estase e dilatação gástrica com diminuição ou aumento de contrações peristálticas, bem como quadros gástricos mais graves como a gastroparesia cujos sintomas, muitas vezes não diagnosticados, se caracterizam por anorexia, perda de peso, náuseas e vômitos (CLEMENTS; BELL, 1982). A etiologia dessas modificações não é totalmente conhecida, mas alterações degenerativas do sistema nervoso entérico (SNE) têm sido relacionadas com a neuropatia diabética. 


\section{Sistema nervoso entérico}

O SNE consiste em uma rede neural presente na parede do trato gastrintestinal do esôfago até o ânus, composto por aproximadamente $10^{8}$ neurônios sensoriais, motores e interneurônios, distribuídos nos dois plexos principais, o mioentérico e o submucoso. Embora espacialmente separados a conexão entre os dois sugere que eles compreendem uma unidade integradora (COSTA et al., 1980; FURNESS, 2005). Tais neurônios controlam a motilidade intestinal e a secreção, iniciando reflexos em resposta ao conteúdo luminal e a tensão do músculo liso (FURNESS; KUNZE; CLERC, 1999; FURNESS, 2012).

Os neurônios que liberam óxido nítrico (NO) em suas terminações nervosas, chamados de neurônios nitrérgicos (nNOS), compõem uma subpopulação proporcionalmente importante do SNE. Estes possuem uma função inibitória crucial no controle da motilidade do trato gastrintestinal (BULT et al., 1990; JARVINEN et al., 1999). Considerando que o óxido nítrico é o maior neurotransmissor inibitório não adrenérgico e não colinérgico do TGI, sua alteração, em condições patológicas como no diabetes, pode estar relacionada a sinais e sintomas normalmente encontrados no trato digestório.

Outra subpopulação neuronal importante corresponde aos VIPérgicos (VIP) que respondem ao diabetes com alargamento das varicosidades e aumento da intensidade de fluorescência, culminando em deterioração do transporte axonal (BELAI; BURNSTOCK, 1990; ZANONI et al., 2002). VIP juntamente com o NO compreende um importante neurotransmissor inibidor não adrenérgico e não colinérgico (CHANDRASEKHARAN; SRINIVASAN, 2007). Desse modo, o comprometimento da liberação de VIP pode contribuir para a motilidade alterada do intestino em animais diabéticos (BALLMANN; CONLON, 1985). Além do mais, neurônios que expressam VIP são conhecidos por desempenhar várias outras funções, tais como na neuroproteção e em processos inflamatórios (EKBLAD; BAUER, 2004) além de possuírem papel neurotrófico (MASMOUDI-KOUKI et al., 2007).

Os neurônios ganglionares e os seus feixes de fibras nervosas no SNE são providos por numerosas células gliais (Furness, 2005). Tem sido bem estabelecido que estas células desempenham um papel relevante na fisiologia e fisiopatologia do TGI (RÜHL; NASSER; SHARKEY, 2004; RUHL, 2005). Este papel está relacionado com sua atuação na homeostase do intestino, bem como serve de elo entre sistema nervoso e imunológico e ainda, atua no controle do fenótipo neuroquímico (AUBÉ et al., 2006). Embora o papel dos neurônios entéricos no DM tem sido amplamente estudado, a contribuição da glia entérica é pouco conhecida.

Diabetes e degeneração neuronal no sistema nervoso entérico

O efeito do DM na população de neurônios entéricos é frequentemente estudado em animais roedores induzidos por estreptozootocina (STZ) ao DM tipo I. A seguir, algumas evidências da degeneração neuronal já encontradas:

\section{Alterações no número e tamanho de neurônios}

Diversos estudos reportam uma redução numérica e concomitante aumento no tamanho do corpo celular em neurônios de vários segmentos do trato gastrointestinal de animais diabéticos induzidos por STZ, tais como: estômago (FREGONESI et al., 2001), duodeno (BÜTTOW; MIRANDA NETO; BAZOTTE, 1997; LOPES et al., 2012), jejuno (HERMES-ULIANA et al., 2013) íleo (HERNANDES et al., 2000; ZANONI et al., 2003; PEREIRA et al., 2011), colo proximal (ROMANO et al., 1996; ROMANO; MIRANDA NETO; CARDOSO, 1996) e ceco (ZANONI et al., 1997; FERREIRA et al., 2013). Redução similar no número de neurônios também tem sido observado em ratos espontaneamente diabéticos (CHANDRASEKHARAN et al., 2011). Alterações estruturais degenerativas como hipertrofia axonal tem sido observado no início e duas semana após o começo do DM (DIANI et al., 1976). Além disso, estudos mostraram que no gânglio da raiz dorsal os neurônios com diâmetro maiores são mais susceptíveis a injúria quando comparado com neurônios menores (KISHI et al., 2002). Ainda, de acordo com Chandrasekharan e Sirinivasan (2007), estas alterações no DM são frequentemente observadas em duas fases distintas: uma fase inicial de perda de neurônios e uma tardia com uma possível regeneração.

\section{Alterações no código neuroquímico dos neurônios}

No sistema nervoso entérico, os neurônios exibem grande variedade na expressão de determinados neurotransmissores, que por sua vez, determinam seu código químico e sendo que muitos estudos descrevem alterações neste código mediante a neuropatia diabética (CHANDRASEKHARAN; SRINIVASAN, 2007; YARANDI; SRINIVASAN, 2014). Existem evidências de que as subpopulações neuronais respondem diferentemente ao diabetes, algumas exibem degeneração, outras alteram seu código neuroquímico sem ocorrer degeneração e ainda, outras não são afetadas (BELAI; BURNSTOCK, 1990; CHANDRASEKHARAN; SRINIVASAN, 2007; FERREIRA et al., 2013). Belai e Burnstock (1991) estudando os efeitos do diabetes induzido por STZ em nervos mioentéricos adrenérgicos e peptidérgicos no íleo e no colo distal de ratos, verificaram redução no nível de noradrenalina e elevação significativa do neuropeptídeo VIP no íleo. Comparado a este segmento, o colo distal teve sua inervação adrenérgica e peptidérgica menos afetada. Outras análises também evidenciaram alterações nos níveis de vários neurotransmissores nos neurônios mioentéricos (BALLMANN; Conlon, 1985), sugerindo que a neuropatia decorrente do DM não é seletiva e compromete o plexo mioentérico como um todo (BELAI et al., 1991).

\section{Balanço de neurônios inibitórios, excitatórios e sensoriais}

A plasticidade e remodelação neuronal entérica que ocorre no DM afeta a taxa de neurônios inibitórios e excitatórios, o qual leva a alterações na motilidade intestinal. Dados sugerem que neurônios inibitórios como nNOS, VIP, e os que produzem o neuropeptídeo $\mathrm{Y}$ e galanina são mais severamente afetados na neuropatia diabética que os excitatórios, entre eles os neurônios acetilcolina transferase (ChAT) 
e os que contém a substância P (CHANDRASEKHARAN; SRINIVASAN, 2007). Adicionalmente, alguns estudos reportam que a densidade de neurônios colinérgicos aumenta no jejuno e íleo de animais diabéticos, acarretando aumento na contratilidade do intestino (CHANDRASEKHARAN; SRINIVASAN, 2007; MILLER et al., 2008; YARANDI; SRINIVASAN, 2014). Assim, esse desequilíbrio entre redução da influência inibitória e aumento da contratilidade pode ser a gênese de muitos sintomas observados no DM, entre eles a diarreia (ANITHA et al., 2006; IZBEKI et al., 2008). Da mesma forma, neurônios sensoriais também são afetados. Entre estes, neurônios que possuem o gene da calcitonina foram encontrados ser significativamente reduzidos no íleo e colo de ratos após oito semanas de DM (BELAI et al., 1996; SPANGEUS, SUHR; EL-SALHY, 2000). Outros como os neurônios que contém o polipeptídio denominado de substância $\mathrm{P}$, responsável pela transmissão da dor, também reduziram em densidade no estômago e íleo de ratos com 5 até 44 semanas de DM.

\section{Patogênese da neuropatia diabética entérica}

O aumento do estresse oxidativo e as alterações na capacidade antioxidante, observadas no estado diabético, tanto em condições clínicas como experimentais, têm sido implicadas no desenvolvimento da neuropatia e de outras complicações típicas dessa doença (CAMERON, COTTER; MAXFIELD, 1993; VAN DAM et al., 1998; LIM et al., 2001; SANDERS, RAUSCHER; WATKINS, 2001). O estresse oxidativo ocorre quando há um desequilíbrio entre a produção de oxidantes e o respectivo sistema de defesa do organismo (ABUJA; ALBERTINI, 2001), levando ao aumento de moléculas reativas ao oxigênio (radicais livres) dentro das células (PARTHIBAN et al., 1995; OBROSOVA et al., 2002). As principais fontes endógenas geradoras de substâncias reativas ao oxigênio incluem as mitocôndrias, nas quais pode ocorrer a redução incompleta do oxigênio na cadeia respiratória além da atividade de enzimas como xantina oxidase, citocromo P450-oxidase, monoaminooxidases, ciclooxigenases, lipooxigenases e a NADPH-oxidase (ABUJA; ALBERTINI, 2001; ROVER JUNIOR; HÖEHR; VELLASCO, 2001). Na natureza, entre as substâncias geradoras de radicais livres encontram-se o oxigênio no estado fundamental $\left(\mathrm{O}_{2}\right)$ e o óxido nítrico $(\mathrm{NO})$, esse último, além de ser sintetizado em diversas células, é encontrado como poluente atmosférico (ROVER JUNIOR; HÖEHR; VELLASCO, 2001).

$\mathrm{Na}$ neuropatia e em outras complicações características do DM, o estresse oxidativo é intensificado pela redução nos níveis das enzimas que participam do sistema de defesa antioxidante como, por exemplo, superóxido dismutase (PARTHIBAN et al., 1995), glutationa peroxidase, glutationa redutase e catalase (OBROSOVA et al., 2002) e, também, pela redução nos níveis de antioxidantes como o ácido ascórbico (YOUNG; TORNEY; TRIMBLE, 1992), a glutationa e a vitamina E (LEE; CHUNG, 1999). Outro mecanismo potencial para o aumento do estresse oxidativo no DM é o aumento desproporcional da formação de radicais livres em decorrência da oxidação da glicose, da glicação não enzimática das proteínas e subsequente degradação oxidativa das proteínas glicadas (MARITIM; SANDERS;
WATKINS, 2003) da micro e macroangiopatia relacionada à hipóxia, de alterações nos níveis de mediadores inflamatórios e na atividade da via do poliol (BAYNES, 1991; DAVISON et al., 2002).

A hiperglicemia ativa a aldose redutase e a sorbitol desidrogenase, enzimas da via do poliol, que catalisam a conversão da glicose em sorbitol e frutose, respectivamente. Como as enzimas frutase e fosfo-frutase, que participam da metabolização do sorbitol e da frutose, apresentam uma atividade funcional limitante, e como o sorbitol e a frutose não se difundem facilmente pela membrana plasmática, ocorre acúmulo gradual de sorbitol e de frutose no interior das fibras nervosas (SILVA; TEIXEIRA, 1999). As consequências deste acúmulo são: 1) aumento da osmolaridade intracelular, com formação de edema, lesão neuronal e redução da condução nervosa (HOSKING; BENNETT, HAMPTON, 1978), 2) redução no conteúdo do mioinisitol, resultando em queda no metabolismo do fosfoinositídeo e menor atividade de diacilglicerol, proteína cinase $\mathrm{C}$ e $\mathrm{Na}+/ \mathrm{K}+$ ATPase (COTRAN; KUMAR; ROBBINS, 1996), levando a uma disjunção axo-glial e danificando a fibra, podendo ser a primeira anormalidade estrutural da neuropatia diabética, e 3) diminuição dos níveis de glutationa pois, tanto a glutationa redutase como a aldose redutase requerem NADPH, havendo, portanto, uma competição pelo mesmo co-fator (CAMERON; COTTER; MAXFIELD, 1993).

Como resultado do aumento da atividade dos radicais livres, a disfunção do tecido nervoso diabético pode ser provocada por efeitos diretos oriundos da peroxidação lipídica das membranas do axônio e das células de Schwann, levando a uma diminuição da função das células nervosas (CAMERON; COTTER; MAXFIELD, 1993), bem como por efeitos indiretos gerados via vascular. Neste caso, as lipoproteínas (LDL), cujas concentrações encontram-se aumentadas no diabetes, quando são oxidadas tornam-se citotóxicas para vários tipos de células, incluindo as células endoteliais, inibindo o relaxamento do tecido vascular (MOREL; CHISOLM, 1989).

\section{Antioxidantes no tratamento da neuropatia do dm}

Múltiplas estratégias terapêuticas demonstram que o tratamento com antioxidantes pode prevenir ou reverter a disfunção nervosa periférica em ratos diabéticos induzidos por estreptozootocina (CAMERON; COTTER; MAXFIELD, 1993; VAN DAM et al., 1998; CAMERON; COTTER, 1999). Tanto a disfunção neurovascular, quanto a condução nervosa deficiente, as alterações metabólicas e os danos oxidativos observados na neuropatia diabética inicial podem ser revertidos pelo tratamento com inibidores da aldose redutase (CAMERON, COTTER; MAXFIELD, 1993; BELAI et al., 1996; OBROSOVA et al., 2002). Sendo assim, substâncias como os antioxidantes que atuam reduzindo o estresse oxidativo e/ou inibindo a aldose redutase, podem ter um papel relevante no tratamento das complicações neurológicas do diabetes. A seguir, alguns dos principais estudos que empregaram antioxidantes no tratamento da neuropatia diabética autonômica no sistema nervoso entérico. 


\section{Vitamina E ( $\alpha$-tocoferol)}

A vitamina E consiste em um potente antioxidante lipofílico (PEREIRA et al., 2008), podendo apresentar-se em oito diferentes formas: $\alpha, \beta, \delta$ e $\gamma$-tocoferol e $\alpha, \beta, \delta$ e $\gamma$-tocotrienol, sendo o $\alpha$-tocoferol a forma biologicamente ativa (DEBIER; LARONDELLE, 2005). O efeito da vitamina $\mathrm{E}$ sob os radicais livres é importante para prevenir ou atenuar muitas doenças degenerativas, entre elas o câncer, doenças inflamatórias, cardiovasculares, envelhecimento e doenças neurológicas (ROLDI et al., 2009). Pesquisadores utilizaram esta substância na dose de 0,1 e $2 \%$ no tratamento da neuropatia entérica do colo proximal de ratos diabéticos por 120 dias e observaram que houve neuroproteção da população total de neurônios mioentéricos bem como redução na hipertrofia do corpo celular (ROLDI et al., 2009). Em contrapartida, Tronchini et al. (2012) utilizando estas mesmas doses de vitamina $\mathrm{E}$ não observaram neuroproteção na população neuronal geral e nitrérgica (nNOS) no jejuno de animais diabéticos, mas observaram hipertrofia do corpo celular neste último grupo de neurônios quando comparado aos animais controle. Similarmente e Pereira et al. (2008), utilizando a dose de $1 \mathrm{~g} / \mathrm{kg}$ de peso corporal não observou diferenças na quantidade de neurônios da população total e nitrérgica no íleo de animais diabéticos, entretanto sugeriu que este composto atuou causando neuroproteção sobre o processo de envelhecimento decorrente do tempo experimental do estudo e, adicionalmente, também causaram efeito neurotrófico em neurônios nitrérgicos (nNOS).

\section{Vitamina C (ácido ascórbico)}

A vitamina $\mathrm{C}$ consiste em uma molécula quiral que ocorre naturalmente em alimentos sob duas formas: a forma reduzida (geralmente designada como ácido ascórbico) e a forma oxidada (ácido desidroascórbico) (ANDERSON et al., 1988). Esta vitamina tem sido amplamente usada na prevenção da perda neuronal em condições patológicas como o DM. Entretanto, o limite entre os efeitos benéficos e maléficos da suplementação com esta substância ainda permanecem desconhecidos (FREITAS et al., 2012). No caso de estudos realizados no SNE do duodeno de animais diabéticos, o ácido ascórbico na dose de $50 \mathrm{mg} / \mathrm{kg}$ apresentou efeito neuroprotetor nos neurônios da população NADH-diaforase (PEREIRA; BAGATIN; ZANONI, 2006). No entanto, no íleo esta substância não apresentou efeito sobre a densidade destes neurônios, mas preveniu o aumento na área do corpo celular dos mesmos (ZANONI et al., 2003).

\section{Ginko biloba}

O Ginkgo biloba consiste em um dos suplementos naturais mais vendidos no mundo, extraído da folha da planta Ginkgo biloba. Apresenta propriedades medicinais importantes entre elas sua atividade antioxidante e efeito neuroprotetor, inibindo a morte celular de várias linhagens celulares (CALAPAI et al., 2000; MACLENNAN, DARLINGTON; SMITH, 2002). Seu uso no SNE do jejuno e íleo na dose de $50 \mathrm{mg} / \mathrm{kg}$ de peso corporal, demonstrou ser eficiente contra perda neuronal ocasionada pelo DM, evidenciada pela neuroproteção da população geral em ambos os segmentos além da redução na área neuronal destes neurônios avaliados (SILVA; ZANONI; BUTTOW, 2011).

\section{Quercetina}

A Quercetina é o principal representante dos flavonóis, subclasse da família dos flavonoides, sendo amplamente encontrado em vários alimentos, como cebolas, maçãs, brócolis, chá e vinho tinto (LOPES et al., 2012; FERREIRA et al., 2013). Na literatura a Quercetina é conhecida por seus efeitos farmacológicos benéficos sobre os sistemas biológicos, como antiperoxidativo, anticarcinogênico, anti-inflamatório e antioxidantes (FERREIRA et al., 2013). Seu estudo também foi conduzido no SNE do duodeno e ceco, na dose de $200 \mathrm{mg} / \mathrm{kg}$. No duodeno, Lopes et al. (2012) encontraram prevenção da perda neuronal da população geral e da subpopulação nNOS, além de reduzir a hipertrofia do corpo celular destes neurônios. Em adição, estes autores verificaram redução significativa no número de células gliais em animais diabéticos e o uso da Quercetina reverteu esta perda. Paralelamente, a análise realizada no ceco por Ferreira et al. (2013) também mostrou neuroproteção da população geral utilizando a mesma dose de quercetina, mas esta não foi observada na subpopulação nNOS. Além disso, em ambos os trabalhos, verificaram que o tamanho do corpo celular destes neurônios foi aumentado com o uso desta substância. Cabe ressaltar também que em ambos os estudos as varicosidades de neurônios VIP foram avaliadas, e o uso da Quercetina reduziu de forma significativa o tamanho destas tanto no duodeno como no ceco de animais com DM.

\section{L-Glutamina/L-Glutationa}

L-Glutamina é um dos aminoácidos essenciais livres mais abundantes no plasma e tecidos musculares (ZANONI et al., 2011). Atua como substrato para a formação de glutationa, um potente antioxidante endógeno (ALVES et al., 2010). Além disso, o uso da glutamina e glutationa na suplementação da dieta podem aliviar os danos neuronais entéricos causados pelo DM (HERMES-ULIANA et al., 2013). Um estudo realizado por Pereira et al. (2011) evidenciou que a L-Glutamina na dose de $2 \%$ preveniu a redução da população neuronal geral bem como promoveu redução na população de células gliais e aumento na área celular, sugerindo ação neuroprotetora e glioprotetora, respectivamente. Outro estudo, utilizando a dose de 1\% demonstrou ação neuroprotetora no duodeno e concomitante redução da hipertrofia do corpo celular, mas estes efeitos não foram observados no ceco de animais com DM (ZANONI et al., 2003). Em adição, nesta mesma dose, Tashima et al. (2007) observaram neuroproteção da população geral no cólon proximal de ratos diabéticos, mas a L-Glutamina não foi capaz de reduzir a área celular destes neurônios. Empregando a dose de 2\% desta substância, Alves et al. (2010) verificaram redução significativa da área do corpo celular e das varicosidades no jejuno de animais com DM. Finalmente, Hermes-Uiliana et al. (2013) empregando a mesma dose de $2 \%$ de L-Glutamina também verificou neuroproteção na população total do jejuno, no entanto, essa foi maior quando os animais diabéticos foram suplementados com $2 \%$ de L-Glutationa, sugerindo que esta última substância é mais promissora que a L-glutationa para 
reduzir a neuropatia diabética entérica.

\section{Aminoguanidina}

A Aminoguanidina tem sido utilizada como um tratamento potencial para a neuropatia diabética, principalmente devido as suas funções como inibidores da formação de produtos finais de glicação avançada (CAMERON et al., 1992), inibidor da aldose redutase (KUMARI et al., 1991) e óxido nítrico sintase (JIANMONGKOL et al., 2000) além de atuar como antioxidante (COURDEROT-MASUYER et al., 1999). Seus efeitos neuroprotetores foram avaliados no íleo de ratos diabéticos (SHOTTON; ADAMS; LINCOLN, 2007) e o tratamento com esta substância na dose de $0,9 \mathrm{~g} / \mathrm{L}$ apesar de aumentar a atividade da enzima nNOS e reduzir o tamanho das varicosidades VIP, não foram igualmente eficaz para todos os nervos autônomos, necessitando novas investigações baseadas em novas doses de tratamento.

\section{Considerações Finais}

A análise da literatura nos leva a concluir que a neuropatia diabética autonômica gastrintestinal acarreta prejuízos na função gástrica (sensorial e motora) e anormalidades na secreção hormonal dos órgãos do sistema digestivo, podendo estar relacionados diretamente com as alterações no SNE. Nesse contexto, muitos autores demonstram que o estresse oxidativo, decorrente da hiperglicemia do DM, é um mecanismo potencial para estes achados. Na maioria das pesquisas com antioxidantes, o uso desses mostraram-se terapias promissoras para prevenir as alterações morfoquantitativas comumente encontradas em neurônios e células gliais de roedores com DM induzidos por estreptozootocina. Entretanto, novas dosagens bem como a eficácia quanto à toxicidade no uso destas substâncias carecem de maiores investigações, tornando-se um tema aberto para novas investigações em diversas áreas, tais como na pesquisa clínica, morfológica, farmacológica, entre outras.

\section{Referências}

ABUJA, P. M.; ALBERTINI, R. Methods for monitoring oxidative stress, lipid peroxidation and oxidation resistance of lipoproteins. Clin Chim Acta, v. 306, n. 1-2, p. 1-17, Apr. 2001.

ALVES, E. P. et al. Immunohistochemical study of vasoactive intestinal peptide (VIP) enteric neurons in diabetic rats supplemented with L-glutamine. Nutr Neurosci. v. 13, n. 1, p. 43-51, Feb. 2010.

ANDERSON, L. et al. Nutrição. 17. ed. Rio de Janeiro: Guanabara Koogan, 1988.

ANITHA, M. et al. GDNF rescues hyperglycemia-induced diabetic enteric neuropathy through activation of the PI3K/ Akt pathway. J Clin Invest, v. 116, n. 2, p. 344-56, Feb. 2006.

ARAÚJO, J. Neuropatia autonômica. Rev. Bras. Neurologia, n. 32, p. 207-209, 1996.
ASSOCIATION, A. D. Diagnosis and classification of diabetes mellitus. Diabetes Care, v. 31 Suppl 1, p. S55-60, jan. 2008 .

jan. 2011.

Diabetes Care, v. 34 Suppl 1, p. S62-9,

AUBÉ, A. C. et al. Changes in enteric neurone phenotype and intestinal functions in a transgenic mouse model of enteric glia disruption. Gut, v. 55, n. 5, p. 630-7, May, 2006.

ALLMANN, M.; CONLON, J. M. Changes in the somatostatin, substance $\mathrm{P}$ and vasoactive intestinal polypeptide content of the gastrointestinal tract following streptozotocin-induced diabetes in the rat. Diabetologia, v. 28 , n. 6, p. 355-8, Jun. 1985.

BAYNES, J. W. Role of oxidative stress in development of complications in diabetes. Diabetes, v. 40, n. 4, p. 405-412, Apr. 1991.

BAZZOTE, R. Paciente diabético: cuidados farmacêuticos. Rio de Janeiro: MedBook, 2010.

BELAI, A.; BURNSTOCK, G. Changes in adrenergic and peptidergic nerves in the submucous plexus of streptozocindiabetic rat ileum. Gastroenterology, v. 98, n. 6, p. $1427-$ 1436, Jun. 1990.

BELAI, A. et al. Enteric neuropeptides in streptozotocindiabetic rats; effects of insulin and aldose reductase inhibition. J Auton Nerv Syst, v. 58, n. 3, p. 163-9, May 1996.

BELAI A. Differential effect of streptozotocin-induced diabetes on the innervation of the ileum and distal colon. Gastroenterology, v. 100, n. 4, p. 1024-32, Apr. 1991.

BONNEFONT-ROUSSELOT, D. Glucose and reactive oxygen species. Curr Opin Clin Nutr Metab Care, v. 5, n. 5, p. 561-568, Sep. 2002.

BULT, H. et al. Nitric oxide as an inhibitory non-adrenergic non-cholinergic neurotransmitter. Nature, v. 345, n. 6273, p. 346-347, May 1990.

BYTZER, P. et al. Prevalence of gastrointestinal symptoms associated with diabetes mellitus: a population-based survey of 15,000 adults. Arch Intern Med. v. 161, n. 16, p. 19891996, Sep. 2001.

BÜTTOW, N. C.; MIRANDA NETO, M. H.; BAZOTTE, R. B. Morphological and quantitative study of the myenteric plexus of the duodenum of streptozotocin-induced diabetic rats. Arq Gastroenterol, v. 34, n. 1, p. 34-42, jan./mar. 1997.

CALAPAI, G. et al. Neuroprotective effects of Ginkgo biloba extract in brain ischemia are mediated by inhibition of nitric oxide synthesis. Life Sci. v. 67, n. 22, p. 2673-83, Oct. 2000. 
CAMERON, N. E.; COTTER, M. A. Effects of antioxidants on nerve and vascular dysfunction in experimental diabetes. Diabetes Res Clin Pract, v. 45, n. 2-3, p. 137-146, Sep. 1999.

CAMERON, N. E. et al. Effects of aminoguanidine on peripheral nerve function and polyol pathway metabolites in streptozotocin-diabetic rats. Diabetologia, v. 35, n. 10, p. 946-50, Oct. 1992.

CAMERON, N. E.; COTTER, M. A.; MAXFIELD, E. $\mathrm{K}$. Anti-oxidant treatment prevents the development of peripheral nerve dysfunction in streptozotocin-diabetic rats. Diabetologia, v. 36, n. 4, p. 299-304, Apr. 1993.

CHANDRASEKHARAN, B. et al. Colonic motor dysfunction in human diabetes is associated with enteric neuronal loss and increased oxidative stress. Neurogastroenterol Motil, v. 23, n. 2, p. 131-138, Feb.2011.

CHANDRASEKHARAN, B.; SRINIVASAN, S. Diabetes and the enteric nervous system. Neurogastroenterol Motil, v. 19, n. 12, p. 951-60, Dec. 2007.

CLARKE, B. F.; EWING, D. J.; CAMPBELL, I. W. Diabetic autonomic neuropathy. Diabetologia, n. 17, p. 195-212, 1979.

CLEMENTS, R. S. Jr.; BELL, D. S. Diabetic neuropathy: peripheral and autonomic syndromes. Postgrad Med. v. 71, n. 6, p. 50-67 Jun. 1982

COSTA, M. et al. Immunohistochemical localization of polypeptides in peripheral autonomic nerves using whole mount preparations. Histochemistry, v. 65, n. 2, p. 157-65, Feb. 1980.

COTRAN, R. S.; KUMAR, V.; ROBBINS, S. L. Robbinspatologia estrutural e funcional. 5. ed. Rio de Janeiro: Guanabara Koogan, 1996.

COURDEROT-MASUYER, C. et al. Antioxidant properties of aminoguanidine. Fundam Clin Pharmacol. v. 13, n. 5, p. 535-540, 1999.

DA SILVA, G. G.; ZANONI, J. N.; BUTTOW, N. C. Neuroprotective action of Ginkgo biloba on the enteric nervous system of diabetic rats. World J Gastroenterol, v. 17, n. 7, p. 898-905, Feb. 2011.

DAVISON, G. W. et al. Exercise, free radicals, and lipid peroxidation in type 1 diabetes mellitus. Free Radic Biol Med, v. 33, n. 11, p. 1543-51, Dec. 2002.

DE FREITAS, P. et al. Neuroprotection and neurodegeneration in submucosal VIP-IR neurons in the jejunum of ascorbic acid supplemented aging Wistar rats. Nutr Neurosci. Aug. 2012.

DEBIER, C.; LARONDELLE, Y. Vitamins A and E: metabolism, roles and transfer to offspring. Br J Nutr. v. 93, n. 2, p. 153-74, Feb. 2005.

DIANI, A. R. et al. A study of the morphological changes in the small intestine of the spontaneously diabetic Chinese hamster. Diabetologia, v. 12, n. 2, p. 101-9, May 1976.

EKBLAD, E.; BAUER, A. J. Role of vasoactive intestinal peptide and inflammatory mediators in enteric neuronal plasticity. Neurogastroenterol Motil. v. 16 Suppl 1, p. 1238, Apr. 2004

FARAG, Y. M.; GABALLA, M. R. Diabesity: an overview of a rising epidemic. Nephrol Dial Transplant. v. 26, n. 1, p. 28-35, Jan. 2011.

FERREIRA, P. E. et al. Diabetic neuropathy: an evaluation of the use of quercetin in the cecum of rats. World $\mathbf{J}$ Gastroenterol. v. 19, n. 38, p. 6416-26, Oct. 2013.

FREGONESI, C. E. et al. Quantitative study of the myenteric plexus of the stomach of rats with streptozotocininduced diabetes. Arq Neuropsiquiatr. v. 59, n. 1, p. 50-53, Mar. 2001.

FURNESS, J. B. The enteric nervous system. New York: Churchill Livingstone, 2005.

. The enteric nervous system and neurogastroenterology. Nat Rev Gastroenterol Hepatol. v. 9, n. 5, p. 286-94, May 2012.

FURNESS, J. B.; KUNZE, W. A.; CLERC, N. Nutrient tasting and signaling mechanisms in the gut. II. The intestine as a sensory organ: neural, endocrine, and immune responses. Am J Physiol. v. 277, n. 5 Pt 1, p. G922-8, Nov. 1999.

GREENE, D. A.; WINEGRAD, A. I. In vitro studies of the substrates for energy production and the effects of insulin on glucose utilization in the neural components of peripheral nerve. Diabetes, v. 28, n. 10, p. 878-887, Oct. 1979

HERMES-ULIANA, C. et al. Is L-Glutathione More effective than L-Glutamine in preventing enteric diabetic neuropathy? Dig Dis Sci. Dec. 2013.

HERNANDES, L. et al. Streptozotocin-induced diabetes duration is important to determine changes in the number and basophily of myenteric neurons. Arq Neuropsiquiatr. v. 58, n. 4, p. 1035-1039, Dec. 2000.

HOSKING, D. J.; BENNETT, T.; HAMPTON, J. R. Diabetic autonomic neuropathy. Diabetes, v. 27, n. 10, p. 1043-55, Oct. 1978.

IZBEKI, F. et al. Immediate insulin treatment prevents gut motility alterations and loss of nitrergic neurons in the ileum and colon of rats with streptozotocin-induced diabetes.

Diabetes Res Clin Pract. v. 80, n. 2, p. 192-8, May 2008. 
JARVINEN, M. K. et al. Nitric oxide synthase-containing neurons in the myenteric plexus of the rat gastrointestinal tract: distribution and regional density. Anat Embryol (Berl), v. 199, n. 2, p. 99-112, Feb. 1999.

JIANMONGKOL, S. et al. Aminoguanidine-mediated inactivation and alteration of neuronal nitric-oxide synthase. J Biol Chem. v. 275, n. 18, p. 13370-6, May 2000.

KISHI, M. et al. Morphometry of dorsal root ganglion in chronic experimental diabetic neuropathy. Diabetes, v. 51, n. 3, p. 819-24, Mar. 2002.

KUMARI, K. et al. Monoaminoguanidine inhibits aldose reductase. Biochem Pharmacol. v. 41, n. 10, p. 1527-8, May 1991.

LEE, A. Y.; CHUNG, S. S. Contributions of polyol pathway to oxidative stress in diabetic cataract. Faseb j. v. 13, n. 1, p. 23-30, Jan. 1999.

LIM, S. S. et al. Synthesis of flavonoids and their effects on aldose reductase and sorbitol accumulation in streptozotocin-induced diabetic rat tissues. J Pharm

Pharmacol. v. 53, n. 5, p. 653-68, May 2001.

LIMA, A. Manual da terapêutica, toxicologia e farmacologia clínica. Rio de Janeiro: Guanabara Koogan, 1993.

LOPES, C. R. et al. Neuroprotective effect of quercetin on the duodenum enteric nervous system of streptozotocininduced diabetic rats. Dig Dis Sci. Aug. 2012.

MACLENNAN, K. M.; DARLINGTON, C. L.; SMITH, P. F. The CNS effects of Ginkgo biloba extracts and ginkgolide B. Prog Neurobiol. v. 67, n. 3, p. 235-257, Jun. 2002.

MALONE, J. I. et al. The effect of hyperglycemia on nerve conduction and structure is age dependent. Diabetes, v. 45, n. 2, p. 209-215, Feb. 1996.

MARITIM, A. C.; SANDERS, R. A.; WATKINS, J. B. Diabetes, oxidative stress, and antioxidants: a review. J Biochem Mol Toxicol. v. 17, n. 1, p. 24-38, 2003.

MASMOUDI-KOUKI, O. et al. Role of PACAP and VIP in astroglial functions. Peptides, v. 28, n. 9, p. 1753-60, Sep. 2007.

MILLER, S. M. et al. Distribution of interstitial cells of Cajal and nitrergic neurons in normal and diabetic human appendix. Neurogastroenterol Motil. v. 20, n. 4, p. 349-57, Apr. 2008.

MOREL, D. W.; CHISOLM, G. M. Antioxidant treatment of diabetic rats inhibits lipoprotein oxidation and cytotoxicity. J Lipid Res. v. 30, n. 12, p. 1827-34, Dec. 1989.

OBROSOVA, I. G. et al. An aldose reductase inhibitor reverses early diabetes-induced changes in peripheral nerve function, metabolism, and antioxidative defense. FASEB J, v. 16, n. 1, p. 123-125, Jan. 2002.

OLMOS, P. R. et al. A new physiopathological classification of diabetic neuropathy. Rev Med Chil. v. 140, n. 12, p. 1593-605, Dec. 2012.

OPPENHEIM, R. Fiquei diabético, e agora? 2. ed. São Paulo: Saraiva, 1996.

PALOMBIT, K. et al. Effects of ischemia and reperfusion on subpopulations of rat enteric neurons expressing the P2X7 receptor. Dig Dis Sci. v. 58, n. 12, p. 3429-39, Dec. 2013.

PARTHIBAN, A. et al. Oxidative stress and the development of diabetic complications--antioxidants and lipid peroxidation in erythrocytes and cell membrane. Cell Biol Int. v. 19, n. 12, p. 987-93, Dec. 1995.

PEREIRA, M. A.; BAGATIN, M. C.; ZANONI, J. N. Effects of the ascorbic acid supplementation on NADHdiaphorase myenteric neurons in the duodenum of diabetic rats. Biocell, v. 30, n. 2, p. 295-300, Aug. 2006.

PEREIRA, R. V. et al. Vitamin E supplementation in rats with experimental diabetes mellitus: analysis of myosin- $\mathrm{V}$ and nNOS immunoreactive myenteric neurons from terminal ileum. J Mol Histol. v. 39, n. 6, p. 595-603, Dec. 2008 .

PEREIRA, R V. et al. L-glutamine supplementation prevents myenteric neuron loss and has gliatrophic effects in the ileum of diabetic rats. Dig Dis Sci. v. 56, n. 12, p. 35073516, Dec. 2011.

ROLDI, L. P. et al. Vitamin E (alpha-tocopherol) supplementation in diabetic rats: effects on the proximal colon. BMC Gastroenterol. v. 9, p. 88, 2009.

ROMANO, E. B.; DE MIRANDA NETO, M. H.; CARDOSO, R. C. S. Preliminary investigation about the effects of streptozotocin-induced chronic diabetes on the nerve cell number and size of myenteric ganglia in rat colon. Rev Chil Anat. v. 14, n. 2, p. 139-145, 1996.

ROVER JUNIOR, L.; HÖEHR, N. F.; VELLASCO, A. P. Sistema antioxidante envolvendo o ciclo metabólico da glutationa associado a métodos eletroanalíticos na avaliação do estresse oxidativo. Quim. nova, v. 24, p. 112-119, 2001.

RUHL, A. Glial cells in the gut. Neurogastroenterol Motil, v. 17, n. 6, p. 777-90, Dec. 2005.

RÜHL, A.; NASSER, Y.; SHARKEY, K. A. Enteric glia. Neurogastroenterol Motil, v. 16 Suppl 1, p. 44-9, Apr. 2004.

SAID, G. Diabetic neuropathy--a review. Nat Clin Pract Neurol, v. 3, n. 6, p. 331-40, Jun. 2007. 
SAMSOM, M. et al. Prevalence of delayed gastric emptying in diabetic patients and relationship to dyspeptic symptoms: a prospective study in unselected diabetic patients. Diabetes Care, v. 26, n. 11, p. 3116-22, Nov. 2003.

SANDERS, R. A.; RAUSCHER, F. M.; WATKINS, J. B. Effects of quercetin on antioxidant defense in streptozotocin-induced diabetic rats. J Biochem Mol Toxicol. v. 15, n. 3, p. 143-149, 2001.

SANT'ANA, D. D. M. G. et al. complicações crônicas do diabetes mellitus e avanços em pesquisas sobre os efeitos da neuropatia diabética no tubo digestivo. Arquivos de Ciências da Saúde Unipar, v. 1, n. 1, p. 27-37, 1997.

SHIRPOOR, A. et al. Effect of vitamin E on oxidative stress status in small intestine of diabetic rat. World $\mathbf{J}$ Gastroenterol. v. 13, n. 32, p. 4340-4344, Aug. 2007.

SHOTTON, H. R.; ADAMS, A.; LINCOLN, J. Effect of aminoguanidine treatment on diabetes-induced changes in the myenteric plexus of rat ileum. Auton Neurosci. v. 132, n. 1-2, p. 16-26, Mar. 2007.

SILVA, C. B.; TEIXEIRA, M. J. Neuropatia diabética. São Paulo, v. 78, p. 150-162, 1999.

SIMA, A. A. New insights into the metabolic and molecular basis for diabetic neuropathy. Cell Mol Life Sci. v. 60, n. 11, p. 2445-2464, Nov. 2003.

SPANGEUS, A.; SUHR, O.; EL-SALHY, M. Diabetic state affects the innervation of gut in an animal model of human type 1 diabetes. Histol Histopathol. v. 15, n. 3, p. 739-744, Jul. 2000.

SRINIVASAN, S.; WILEY, J. W. New insights into neural injury, repair, and adaptation in visceral afferents and the enteric nervous system. Curr Opin Gastroenterol. v. 16, n. 1, p. 78-82, Jan. 2000.

TASHIMA, C. M. et al. Diabetic rats supplemented with L-glutamine: a study of immunoreactive myosin- $\mathrm{V}$ myenteric neurons and the proximal colonic mucosa. Dig Dis Sci. v. 52, n. 5, p. 1233-1241, May, 2007.

THURSTON, J. H. et al. Effects of acute, subacute, and chronic diabetes on carbohydrate and energy metabolism in rat sciatic nerve. Relation to mechanisms of peripheral neuropathy. Diabetes, v. 44, n. 2, p. 190-195, Feb. 1995.

TRONCHINI, E. A. et al. Supplementation with $0.1 \%$ and $2 \%$ vitamin $\mathrm{E}$ in diabetic rats: analysis of myenteric neurons immunostained for myosin-V and nNOS in the jejunum.

Arq Gastroenterol. v. 49, n. 4, p. 284-90, Dec. 2012.

VAN DAM, P. S. et al. Nerve function and oxidative stress in diabetic and vitamin E-deficient rats. Free Radic Biol Med. v. 24, n. 1, p. 18-26, Jan. 1998.

VOUKALI, E.; SHOTTON, H. R.; LINCOLN, J. Selective responses of myenteric neurons to oxidative stress and diabetic stimuli. Neurogastroenterol Motil. v. 23, n. 10, p. 964-411, Oct. 2011.

WOLFF, H. Diabetes mélito. São Paulo: Fundo Editorial Procienx, 1970.

YARANDI, S. S.; SRINIVASAN, S. Diabetic gastrointestinal motility disorders and the role of enteric nervous system: current status and future directions. Neurogastroenterol Motil. v. 26, n. 5, p. 611-24, May 2014.

YOUNG, I. S.; TORNEY, J. J.; TRIMBLE, E. R. The effect of ascorbate supplementation on oxidative stress in the streptozotocin diabetic rat. Free Radic Biol Med. v. 13, n. 1, p. 41-46, 1992.

ZANONI, J. N. et al. Evaluation of the population of NADPH-diaphorase-stained and myosin-V myenteric neurons in the ileum of chronically streptozotocin-diabetic rats treated with ascorbic acid. Auton Neurosci. v. 104, n. 1, p. 32-8, Feb. 2003.

ZANONI, J. N. et al. Morphological and quantitative analysis of the neurons of the myenteric plexus of the cecum of streptozotocin-induced diabetic rats. Arq Neuropsiquiatr. v. 55, n. 4, p. 696-702, Dec. 1997.

ZANONI, J. N. et al. Terminal ileum submucous plexus: Study of the VIP-ergic neurons of diabetic rats treated with ascorbic acid. Arq Neuropsiquiatr. v. 60, n. 1, p. 32-37, Mar. 2002.

SHOTTON, H. R.; ADAMS, A.; LINCOLN, J. Effects of L-glutamine supplementation on the myenteric neurons from the duodenum and cecum of diabetic rats. Arq Gastroenterol. v. 48, n. 1, p. 66-71, jan./mar. 2011.

Recebido: $19 / 02 / 2015$ Aceito: $28 / 08 / 2015$ 